\title{
Colaboraciones
}

\section{Clasificación de materiales de construcción}

\author{
VICENTE A. GALVAÑ LLOPIS, Dr. Arquitecto \\ LILIANA PALAIA PEREZ, Dra. Arquitecta \\ Cátedra de Materiales de Construcción. E.T.S. de Arquitectura de Valencia
}

\section{RESUMEN}

El presente trabajo pretende dar una respuesta agil a una demanda profesional como es la clasificación de los Materiales. Para ello analiza los diferentes sistemas de clasificación existentes y crea un sistema operativo de selección de un material de construcción, en función de sus propiedades específicas como elemento de construcción. Por último resume todos estos datos en la creación de una ficha modelo.

\section{SUMMARY}

The present work pretends to give a quick answer to a professional request, which is to classificate building materials. With that purpose this work analyses some of the different existing systems of classification, creating an operating system to choose a building material attending its specific properties as a constuction element.

Finally, all the information about each material is summarized in a preformated fiche.

\section{ORIGEN DEL TRABAJO Y OBJETO DEL MISMO}

Tanto desde el punto de vista didáctico en el campo de la enseñanza de los materiales de construcción, dentro de la Escuela Técnica Superior de Arquitectura de Valencia, como desde el desarrollo de la actividad profesional de la arquitectura, se ha detectado la inexistencia de un sistema de clasificación operativo que permita y favorezca una adecuada selección de un material de construcción para un fin determinado, basando esta selección en las características que los materiales presentan y que los hacen óptimos para formar parte del sistema constructivo aplicable a cada solución de diseño.

Este trabajo se lleva adelante por los profesores de la asignatura de Materiales de Construcción del centro docente antes citado, buscando una aplicación no sólo didáctica sino también en el campo profesional.

Para el planteamiento del trabajo se ha considerado, como objetivo, que la tarea de clasificación y selección de los materiales pudiera realizarse con la ayuda de un ordenador, que permite la consideración de un mayor número de variables en la valoración de un material de construcción.

Han sido entonces los objetivos trazados para el desarrollo de este trabajo:

1. Formar un sistema operativo de selección de un material de construcción en función de sus propiedades específicas como elemento constructivo.

2. Informatizar el sistema a fin de considerar un mayor número de variables que faciliten la tarea de selección.

\section{SISTEMAS DE CLASIFICACION DISPO- NIBLES}

Los sistemas de clasificación disponibles que han sido estudiados para abordar este trabajo, han sido:

a) Sistema SfB.

b) Sistema CDU.

c) Sistema SEDITEC.

d) Otros sistemas.

Incluimos unas breves consideraciones realizadas con respecto a cada uno de ellos.

\section{a) Sistema $S f B$}

Este sistema internacional de clasificación es muy amplio en su concepción, teniendo como interés el asegurar una mejor coordinación entre todos los profesionales involucrados dentro del campo del proyecto y la costrucción de las edificaciones.

Ha sido pensado fundamentalmente para la catalogación y clasificación de documentos de 
distinta naturaleza considerando una clasificación temática.

Para ello se dispone de cinco tablas, utilizándose en cada una de éstas símbolos diferentes que permiten obtener una información codificada.

Los códigos resultan de lectura universal por parte de los distintos usuarios, prescindiendo de las limitaciones del idioma.

El sistema sueco SfB ha sido estudiado y adoptado por otros países, teniendo mayor repercusión la adaptación francesa SI/SfB, y la de Gran Bretaña, Cl/SfB, que ha logrado un mayor avance en sus aplicaciones, habiendo sido elaborada en 1971 la obra "Cl/SfB Proyect Manual" y es precisamente en la que se ha basado esta valoración del sistema SfB.

Destaca como favorable en este sistema la codificación con fines de coordinación y síntesis, siendo un instrumento para el intercambio profesional internacional.

Puede resultar un factor de complejidad la utilización simultánea de las 5 tablas que constituyen el espectro de símbolos a utilizar.

Estas cinco tablas con la información y símbolos que se detallan:

Tabla 0.-Referente al entorno construido y no construido, utilizando cifras sin paréntesis.

Tabla 1.-Referente a los materiales de construcción, utilizando cifras entre paréntesis.

Tabla 2.-Define los materiales según su forma, con símbolos formados por letras en mayúscula.

Tabla 3.-Referente a los materiales según su naturaleza utilizando letras en minúscula y números.

Tabla 4.-Relaciona actividades y operaciones en la costrucción, utilizando letras en mayúscula, definiendo categorías con letras en minúscula y entre paréntesis.

Se dispone de cuatro casillas para reunir la información, de esta forma:

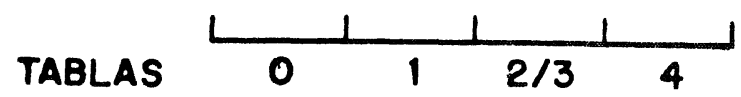

La operatividad del sistema SfB no se pone en duda en cuanto a los objetivos que se han tenido en cuenta para su formación y que son muy distintos a los consideados por los autores del presente trabajo, dirigidos a la selección adecuada de un material de construcción para un fin determinado y teniendo en cuenta sus propiedades.

En todo caso esta selección se realizaría a través del sistema SfB considerando su forma, su naturaleza o por el elemento constructivo que se pretende formar, pero en nada intervendrían en esa operacoín las necesidades creadas en el proyecto de una edificación en su proceso de materialización, ni éste tendría posibilidades de valorar distintas variables.

\section{b) Sistema $C D U$}

Este sistema ideado para el intercambio internacional de publicaciones e información, resulta operativo por la utilización de códigos numéricos.

Creado en 1985 como Clasificación Decimal Dewey, este sistema se ha ido mejorando por la Federación Internacional de documentación FID. En 1950 el Consejo Internacional de Documentación sobre la Construcción CIDB, que se transformó más tarde en el Consejo Internacional de Investigación de la Construcción, Estudios y Documentación CIB, recomendó a sus socios la adopción del CDU para intercambio de información. Se nombró una Junta especial de trabajo para preparar una Clasificación Abreviada de Construcción ABC, pasando más tarde esa junta a formar parte del Comité Internacional de Clasificación de la Construcción, que es un Comité conjunto del CIB y del FID.

Esta clasificación se basa en la adición de cifras que van expresando con más detalle la información.

Siendo el grupo 69 el que se emplea para la documentación referente a la construcción, es el 691 el que define los materiales de construcción a nivel de información general y listas de materiales. Puede ocurrir que determinados aspectos de algunos materiales queden fuera de la agrupación a partir del 691, porque hay que realizar una cuidadosa comprobación de los distintos epígrafes de la relación de material y su código numérico. Por dar un ejemplo, las piedras naturales, y específicamente el granito, quedaría definido por 691.212 y su aspecto de utilización como material de revestimiento, se definiría por 729,6 siendo su referencia completa 691.212.729.6.

Si se refiere a un tipo de publicación se incluye otra cifra entre paréntesis, y si en nuestro ejemplo se quisiera indicar que se tra- 
ta de una publicación periódica, quedaría 691.212.729.6(05).

La ventaja de este sistema, al igual que el anterior, es su universalidad por el empleo de símbolos que originan códigos de interpretación posible por muchas culturas, aunque se escapan de la tarea de selección de los materiales de construcción.

\section{c) Sistema SEDITEC}

Este Servicio de Documentación e Información Técnica Comercial de la Construcción, basa su relación de materiales en el Cl/SfB, adaptado a sus necesidades de localización de productos. Establece grupos de información según partidas relacionadas con el progreso de la obra o gremios que intervienen en el proceso constructivo de una edificación.

En materiales de uso no muy difundido puede establecerse relativa confusión a la hora de acceder al capítulo correspondiente. La información que se recoge consiste en los catálogos comerciales de los distintos productos.

\section{d) Otros sistemas}

Dentro de este grupo se incluyen las clasificaciones que se realizan para determinar las partidas presupuestarias de la construcción de las obras, en base a las tareas que en esa labor intervienen. Son las habituales en la documentación de los proyectos, y que no hacen referencia a los materiales sino a las distintas tecnologías de la construcción.

A modo de ejemplo incluimos una de estas agrupaciones como listado de capítulos:

1. Demoliciones.

2. Red horizontal de saneamiento.

3. Cimentaciones.

4. Estructura.

5. Albañilería.

6. Pinturas.

7. Alicatados y pavimentos cerámicos.

8. Cantería y piedra artificial.

9. Revestimientos y solados.

10. Carpintería interior y exterior.

11. Cerrajería.

12. Vidriería.

13. Fontanería.

14. Electricidad.
15. Red de evacuación.

16. Instalaciones especiales.

17. Varios.

Otro sistema de clasificación, en base a los elementos constructivos, viene determinado por las Normas Tecnológicas de la Edificación (NTE), que sin ser de obligado cumplimiento, sirven de consulta para los profesionales y alumnos de esta actividad.

Cabe mencionar también el sistema internacional de identificación de publicaciones ISBN, International Standard Book Number, formado por la sucesión de cifras hasta el número de diez que permiten identificar el grupo de materia, el editor y el control de la publicación. Igual sentido tiene el ISSN, International Standard Serials Number, aplicable a las publicaciones periódicas.

Existen otras clasificaciones basadas en las expuestas anteriormente, y adaptadas a una aplicación particular pero que en ningún caso ofrecen las características de clasificación perfiladas al encararse este estudio por este equipo de trabajo.

Existe otro sistema de clasificación desarrollado desde hace algún tiempo por la Cátedra de Materiales de Construcción de la Escuela Técnica Superior de Arquitectura de Madrid, a cargo del Profesor Luis de Villanueva, basado en las tablas $2 / 3$ del sistema $\mathrm{Cl} / \mathrm{SfB}$, y en las NTE, dándose prioridad a la clasificación por la naturaleza del material. Quedan por lo tanto los materiales de construcción definidos por su naturaleza, por su forma y por el elemento constructivo que pueden formar. Su aplicación está dirigida a la identificación y archivo de la documentación con referencia a los diferentes materiales.

\section{DESCRIPCION DEL SISTEMA DE CLA- SIFICACION Y SELECCION DE LOS MA- TERIALES, EN PREPARACION}

Resulta prioritario según los objetivos marcados por este equipo de trabajo realizar una selección de un material en función de sus propiedades y posibilidades de aplicación constructiva. Esta cuestión hemos visto que no se atiende en los sistemas anteriormente reseñados, puesto que no era esa su inspiración ni su planteamiento de creación.

Analizando el problema que nos ocupa, ante el vacio existente, se determinaron cinco áreas o conjuntos de información que se pretende abarcar. Estas son:

1. Materiales de construcción. 
2. Normativa básica de caracterización de los materiales.

3. Características de los materiales.

4. Elementos constructivos a los que son aplicables.

5. Localización comercial.

Pasaremos a referirnos con más detalle a cada una de estas áreas o conjuntos de información.

\section{Materiales de construcción}

Surge como punto de partida la ordenación de la primera área, como posible forma de acceso a la información deseada, y se propone para ello realizar una ordenación alfabética de denominación técnica según se defina en su normativa de caracterización a los distintos materiales.

El conjunto de denominación del material está formado por dos subconjuntos: uno de deno- minación técnica ya referido, y otro en base a la denominación comercial con la que en el mercado se lo identifique.

\section{Normativa de caracterización}

Este conjunto está formado por la relación de normas vigentes y recomendaciones, ordenadas según su rango de aplicación, y de la que se desprende así mismo su denominación técnica y el conjunto de propiedades que lo caracterizan, su determinación y aplicación constructiva del material.

\section{Caracterización de los materiales}

Este conjunto de información está formado por cuatro subconjuntos:

3.1. Aspecto.

3.2. Propiedades físicas.

3.3. Propiedades químicas.

3.4. Propiedades mecánicas.

TABLA 1

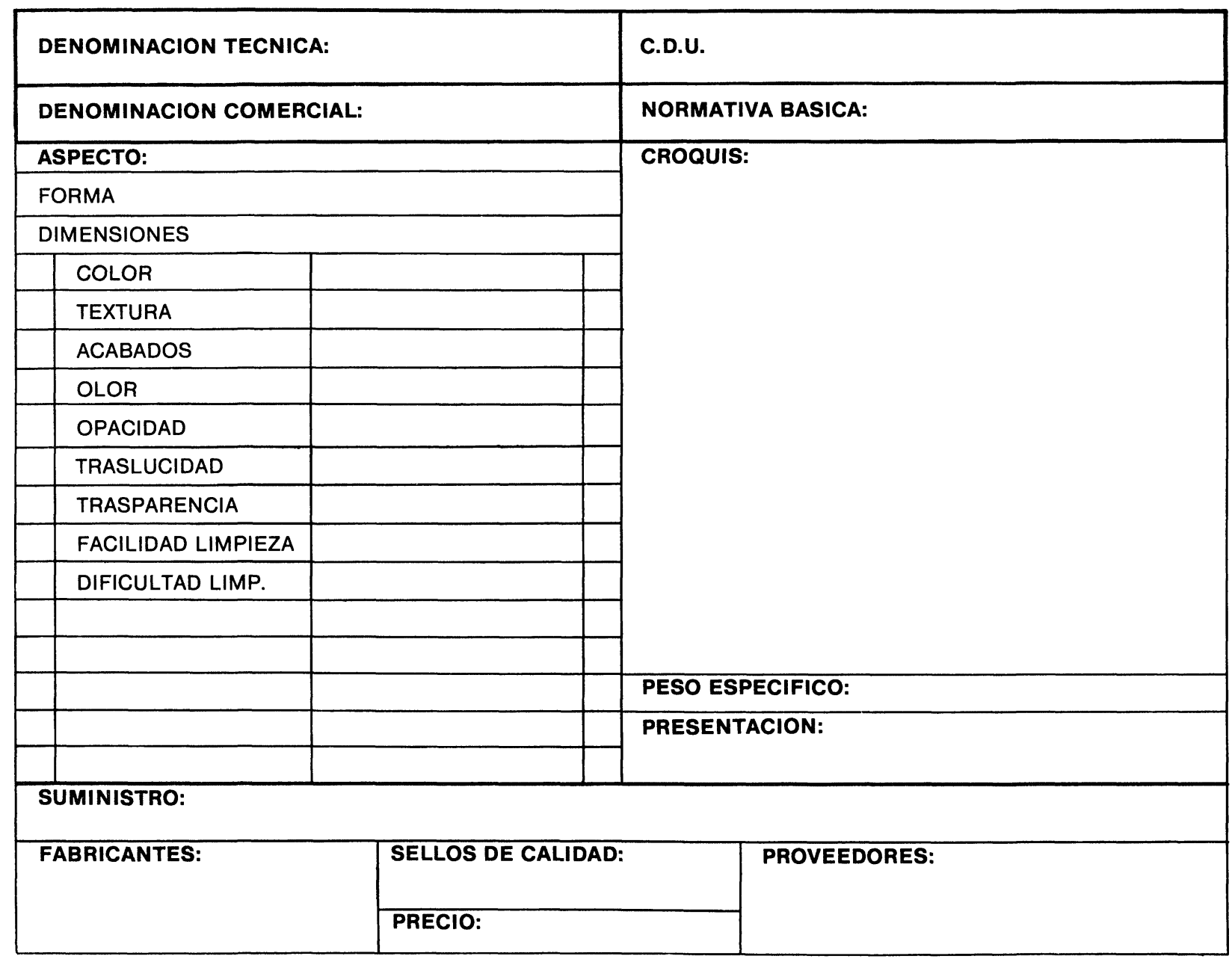


Dentro de cada subconjunto se enumeran, valoran y/o cuantifican estas propiedades en relación al material, reflejando esta valoración sus aptitudes o inconvenientes de adecuación a un uso determinado.

\section{Elementos constructivos}

Para la formación de este conjunto se ha optado por la utilización codificada empleada por las NTE, que si bien se ha indicado que no son de obligado cumplimiento, son de habitual cnsulta y de forma generalizada por parte de alumnos y profesionales dentro de la arquitectura.

Se pretende de esta forma unificar la identifi- cación del elemento constructivo mediante el empleo de estos códigos, sencillos de localizar.

\section{Localización comercial y calidad del material}

Completa la información del material el contenido previsto de este conjunto, formado por cuatro subconjuntos:

- Fabricante.

- Proveedor.

- Sellos de calidad.

- Precio unitario.

TABLA 2

\begin{tabular}{|c|c|}
\hline \multirow{2}{*}{$\begin{array}{l}\text { PROPIEDADES QUIMICAS: } \\
\text { COMPONENTES PRINCIPALES: }\end{array}$} & PROPIEDADES FISICAS: \\
\hline & IGNIFUGO \\
\hline ORGANICO & DENSO \\
\hline INORGANICO & СОМРАСTO \\
\hline SOLUBLE & LIGERO \\
\hline EFLORESCIBLE & POROSO \\
\hline DURABLE ACCION ACIDOS & ABSORBENTE \\
\hline DURABLE ACCION BASES & HELADIZO \\
\hline CORROIBLE & PERMEABLE \\
\hline SOLDABLE & DURABLE INTEMPERIE \\
\hline & ISOTROPO \\
\hline & CONDUCTOR CALOR \\
\hline \multirow[t]{14}{*}{ ELEMENTO CONSTRUCTIVO (NTE) } & ABSORBENTE ACUSTICO \\
\hline & AISLANTE ACUSTICO \\
\hline & CONDUCTOR ELECTRICO \\
\hline & HIGROSCOPICO \\
\hline & HIDROFUGO \\
\hline & PROPIEDADES MECANICAS: \\
\hline & RESISTENTE COMPRESION \\
\hline & RESISTENTE TRACCION \\
\hline & RESISTENTE FLEXION \\
\hline & DURO \\
\hline & FRAGIL \\
\hline & RIGIDO \\
\hline & ELASTICO \\
\hline & PLASTICO \\
\hline \multirow[t]{4}{*}{ OBSERVACIONES: } & TENAZ \\
\hline & DUCTIL \\
\hline & MALEABLE \\
\hline & HENDIBLE \\
\hline
\end{tabular}


A partir de este grupo de información se puede localizar el material una vez seleccionado a la vez que permite derivar su disponibilidad en la zona en que se realiza la obra, y su repercusión económica del mismo dentro del sistema.

\subsection{Ficha resumen}

Toda esta información se implementa a partir de fichas individualizadas por material, en la que se reúnen todos los datos disponibles, cuantificados y valorados. La ficha contiene todos los conjuntos de información previamente referidos, y se completa con un espacio para incluir un croquis acotado del material.

La limitación que puede ofrecer la ordenación alfabética de los materiales se supera mediante la inclusión de su identificación según el sistema CDU, que es, por otra parte, el adoptado por las Normas UNE.

Por último, cada ficha permite recoger aclaraciones orientadas a definición de la procedencia de los datos allí recogidos, bien procedan de catálogos de productos, de normas, de recomendaciones o de ensayos.

\subsection{Alcance y posibilidades del sistema}

La versatilidad del sistema, junto con su posibilidad de informatizarlo, han sido los requisitos operativos para su formulación.

Partiendo de las fichas resumen se puede instrumentar un programa que facilite la selección, vinculando los conjuntos de información entre sí. Este programa ha de permitir acceder al sistema de clasificación de materiales desde al menos los cuatro primeros conjuntos que se han enunciado de manera indistinta, atendiendo a la casuística y circunstancias que se reúnen a la hora de elegir un material.

La elección se prevé que se realice de entre varios materiales que puedan cumplir con los requerimientos de proyecto, teniendo que perfilarse y definirse la opción última en base a cuestiones referentes a disponibilidad del material, su repercusión económica, y a criterios estéticos que no han intervenido como variables en el proceso de selección en una primera aproximación al problema, sino que forman parte de un segundo proceso en el que la persona interesada ya opta por el que mejor atienda al problema planteado.

\section{publicación del i.e.t.c.c.}

\section{ACUEDUCTOS ROMANOS EN ESPAÑA Carlos Fernández Casado \\ Prof. Dr. Ing. de Caminos, Canales y Puertos}

Esta publicación se compone de una serie de articulos, publicados en la Revista "Informes de la Construcción", en los cuales se hace un análisis de los acueductos romanos que existen en España y el balance de las condiciones de conservación en que se encuentra cada uno de ellos, incluyendo referencias históricas y literarias. Se ha ilustrado con la reproducción de la valiosa documentación gráfica que posee el prestigioso autor.

Un volumen encuadernado en couché, a dos colores, de $21 \times 27$ centímetros, compuesto de 238 páginas, numerosos grabados, dibujos, fotos en blanco y negro y figuras de linea.

Precio: España, 900 ptas., 13 \$ USA.

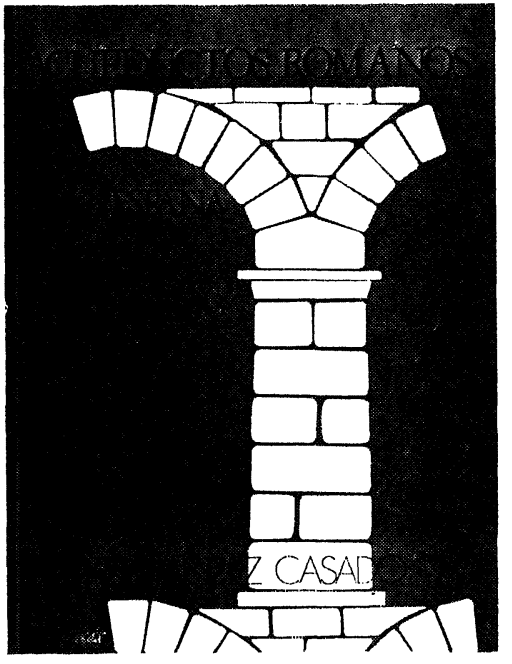

